\title{
Sixteen Years of Trastuzumab Use: Complete Response in Lung, Bone, and CNS Metastasis from Breast Cancer
}

Maria de Lourdes Lopes de Oliveira and Gabriela Passos

Oncology D’Or Department, Rio de Janeiro 22271110, Brazil

\begin{abstract}
Although metastatic breast cancer is considered as an incurable disease, various biological drivers influence the outcomes. The use of trastuzumab in patients overexpressing HER(human epidermal growth factor receptor 2)-2 increases long-term survival even in those patients who developed brain metastasis. Nevertheless, special attention must be paid to the risk of cardiotoxicity. We report the case of a young woman with HER-2-positive breast cancer with bone and lung disease who developed brain metastasis during treatment with trastuzumab. The treatment has been continued and she is alive and in complete remission after 16 years.
\end{abstract}

Key words: Trastuzumab, long-term survival, brain metastasis, complete response, breast cancer.

\section{Introduction}

Breast cancer is a heterogeneous disease with many different clinical expressions. The HER-2-positive phenotype is present in approximately $20 \%$ of invasive breast cancer cases, and HER-2 protein overexpression is associated with poor clinical outcomes. In the 1980s, Slamon and colleagues proposed the use of HER-2 protein overexpression as a guide for therapeutical choices. In 1988, trastuzumab was incorporated into the treatment of metastatic breast cancer [1].

Trastuzumab is a humanized monoclonal antibody that selectively binds to HER-2 on the surface of tumor cells [2]. When used in first-line treatment protocols of metastatic breast cancer in combination with chemotherapy, trastuzumab significantly improves time to progression (TTP, 7.4 vs. 4.6 months, $p<0.001$ ), overall response rate (ORR, $50 \%$ vs. $32 \%$, $p<0.001$ ), and overall survival (OS, 25.1 vs. 20.3 months, $\mathrm{p}=0.046)[3,4]$. As survival in breast cancer patients is improving, brain metastases are increasingly prevalent: $10-30 \%$ of patients with metastatic breast cancer developed brain metastasis

Corresponding author: Maria de Lourdes Lopes de Oliveira, professor in oncology, research fields: medical oncology. during their disease [5]. Lower et al. [6] showed that patients on trastuzumab therapy who developed brain metastases experienced better survival than patients with brain metastases who were not treated with trastuzumab. Within the trastuzumab-treated group, survival was not affected by the presence of brain metastases. In addition, those patients receiving trastuzumab were unlikely to develop new bone metastases [6].

So-called "exceptional responders" are cancer patients with long-term survival who responds to treatment to an unexpected degree. Several studies have attempted to determine the prognostic factors shared by these patients.

We present a case of a patient with metastatic breast cancer to the lung, bones, and brain who has been receiving trastuzumab for 16 years and who has achieved complete response.

\section{Case Presentation}

In December 1996, a 45 year-old woman diagnosed with grade III multicentric ductal carcinoma $(6.0 \times 4.0$ $\mathrm{cm}$ and $2.5 \times 2.0 \mathrm{~cm}$ ) with lymphovascular invasion and 18 positive nodes out of 22 nodes dissected underwent a left mastectomy with axillary dissection. The immunohistochemistry profile was negative for 
hormone receptors.

The patient received adjuvant therapy with four cycles of anthracycline-based chemotherapy followed by eight cycles of CMF (cyclophosphamide + methotrexate + 5-fluouracil) and adjuvant local radiotherapy.

In October 1999, the patient complained of persistent cough and bone pain. A bone scan and a chest CT were performed and revealed bone and lung metastasis (Fig. 1).

At the time, a new chemotherapy protocol was proposed, but the patient declined due to the risk of alopecia. The patient received CNF combination therapy (cyclophosphamide + mitoxantrone + 5-fluouracil) associated with bisphosfonates. After six cycles of treatment, radiology showed that the patient had stable disease.

In March 2000, the HER-2/neu status was determined by FISH (fluorescence in situ hybridization) and confirmed HER-2 overexpression. The patient agreed to change the treatment and trastuzumab was associated with paclitaxel. The paclitaxel therapy was interrupted in June 2000 and the trastuzumab treatment was continued.

In October 2000, a thoracic CT scan showed no evidence of lung metastasis (Fig. 2).

In April 2001, the patient reported dizziness and headache. A brain MRI (magnetic resonance imaging) showed brain metastasis (Figs. 3 and 4).

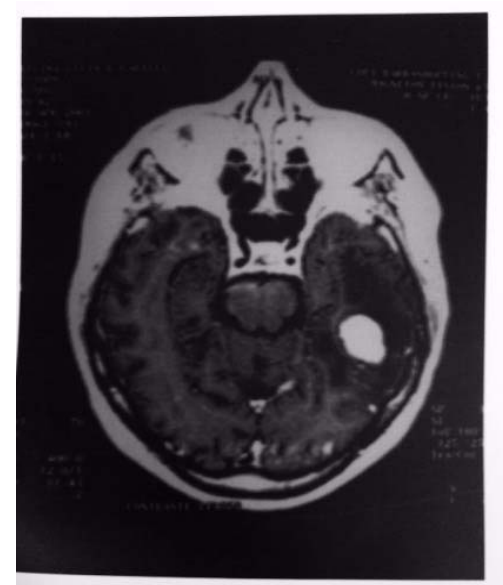

The patient was treated with WBRT (whole-brain radiotherapy) and trastuzumab was reinitiated one month after WBRT was terminated. Six months later, an MRI showed no evidence of radiological disease (Figs. 5 and 6). Since then, the patient has continued receiving trastuzumab at 21-day intervals and cardiac function has been assessed with echocardiography four times a year, with no signs of cardiotoxicity.

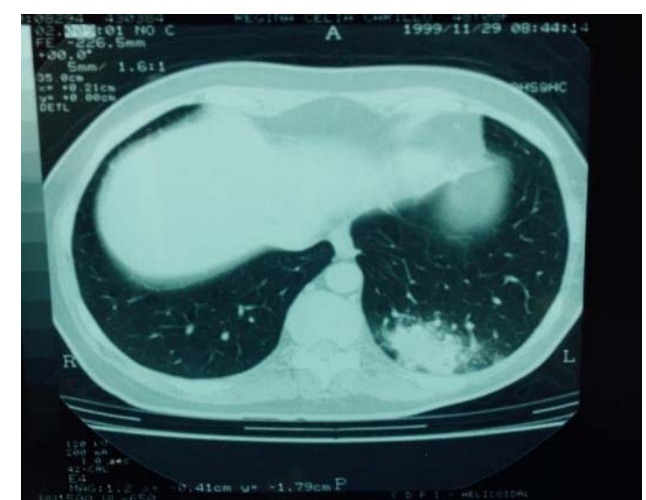

Fig. 1 Lung metastasis.

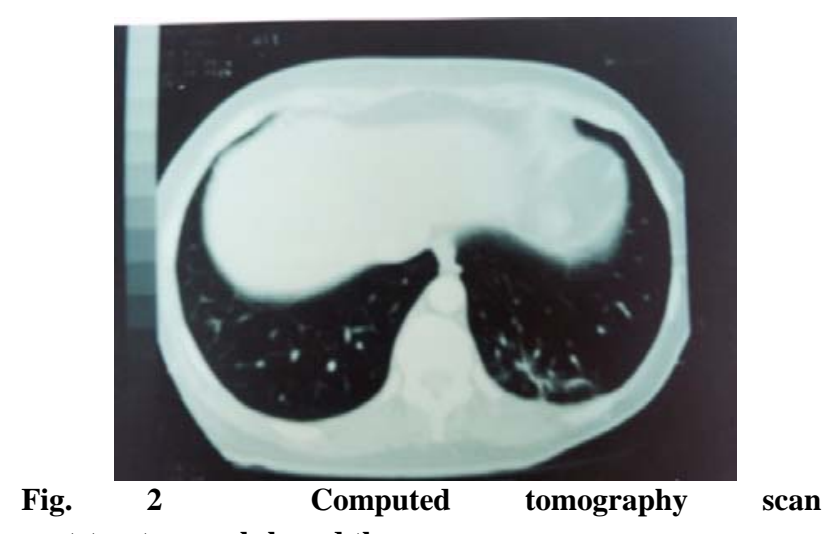
post-trastuzumab-based therapy.

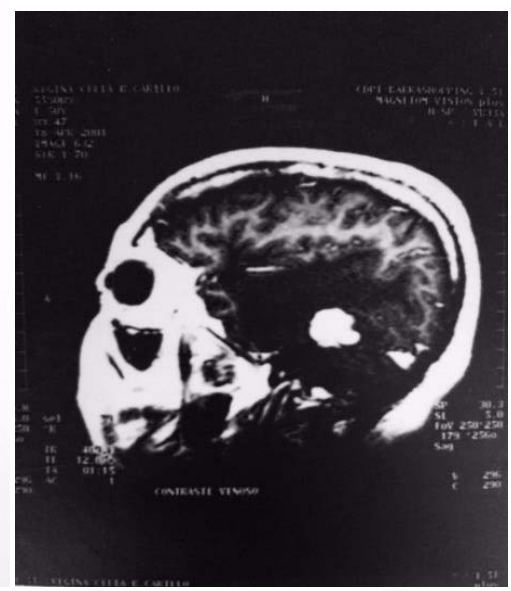

Fig. 3 and 4 Brain magnetic resonance imaging showing metastasis. 

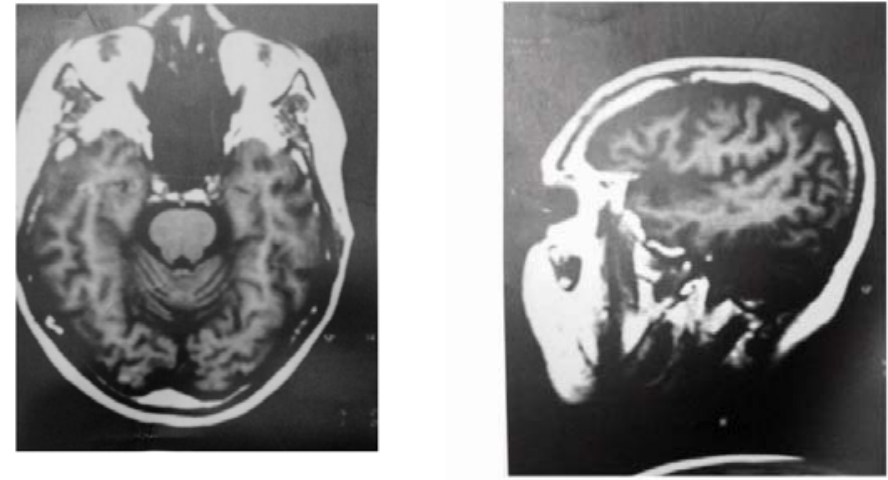

Figs. 5 and 6 Brain magnetic resonance imaging after radiotherapy.

\section{Discussion}

Here, we report the case of a premenopausal woman with breast cancer who developed bone and lung metastases two years after starting adjuvant chemotherapy. During later therapy with trastuzumab, the patient developed brain metastases and received external cranial radiotherapy, and has continued receiving trastuzumab treatment for the last 16 years.

Several studies have attempted to determine the prognostic factors associated with long-term survival in patients with metastatic breast cancer. An observational study that followed 1,023 patients with HER-2-positive metastatic breast cancer reported, as factors associated with increased overall survival, ER receptor-positive or PR receptor-positive metastases (either to bone only or to bone and breast or node/local sites), first-line trastuzumab use, and first-line taxane use [7]. In contrast, the patient reported here had hormone-receptor-negative breast cancer, which evolved to bone and local disease, and received other treatment before switching to taxane and trastuzumab, albeit for less than six months.

A 2016 retrospective study of 168 patients who received trastuzumab therapy in the metastatic setting assessed factors associated with long-term survival. The median overall survival from the time of breast cancer recurrence was 3.9 years. From the time of diagnosis of metastatic disease, $33 \%$ of the patients survived more than five years and only $7 \%$ survived more than 10 years. Of the patients diagnosed with CNS (central nervous system) metastases, 14\% survived more than five years following the diagnosis. Favorable prognostic factors included younger age at diagnosis, lower stage, hormonal receptor positive status and only one organ involved at diagnosis [8].

The risk for developing brain metastases in patients receiving trastuzumab-based therapy for metastatic breast cancer with HER-2 overexpression is $25-40 \%$, compared with $10-30 \%$ in patients not expressing HER-2 [9]. In a study in newly diagnosed HER-2-positive breast cancer patients, the risk factors for developing CNS metastases were young age, African-American ethnicity, ER-negativity, high tumor grade, and BRCA1 phenotype [10].

The patient reported here received WBRT to treat the brain metastasis and showed complete radiological response. The RTOG (Radiation Therapy Oncology Group) published a prognostic classification analysis of patients with breast cancer and brain metastases based on the following clinical factors: Karnofsky performance status, age, and control of extra-cerebral disease [11]. Several reports suggest that HER-2-positive patients who have brain metastases and receive trastuzumab far better than HER-2-negative or HER-2-positive patients who do not receive trastuzumab [12]. The survival advantage may be due to an antitumor activity from trastuzumab at extracranial sites, and to the fact that EBRT (external brain radiotherapy) for brain metastases can 
disrupt the blood-brain barrier allowing trastuzumab entrance into CNS [13]. These factors could explain the better outcome in patients who received trastuzumab beyond progression. Altogether, these factors make it plausible that the survival of the patient is due to the fact that she was young at diagnosis, had good performance status, had other sites of metastasis under control, received cranial radiotherapy, and that trastuzumab treatment was maintained.

Cardiac safety is a point of concern in using trastuzumab for such a long time. The degree of trastuzumab cardiotoxicity ranges from asymptomatic left ventricular dysfunction, which can be diagnosed during routine follow-up, to severe symptomatic heart failure [14]. The NCCTG (Alliance) N9831 trial compared the long-term cardiac safety of trastuzumab used in the adjuvant setting. The cumulative incidence of cardiac events was slightly higher with the addition of trastuzumab in patients receiving taxane or doxorubicin combination regimens, but the authors concluded that trastuzumab continues to have a favorable benefit-risk ratio [15]. The patient reported here was young, had no comorbidities such as hypertension or diabetes, and had received mitoxantrone for a short time. Her cardiac function has been assessed by echocardiography every four months and showed no signs of deterioration.

\section{Conclusions}

Overall survival rates have been dramatically improved since the approval of trastuzumab for patients with metastatic breast cancer overexpressing HER-2.

Several cases of prolonged survival with long-term use of trastuzumab have been reported in the medical literature, none of which trastuzumab was administered for longer than nine years [15].

Even though CNS progression is frequent in trastuzumab-treated patients, there is a benefit, in terms of overall survival, for patients who receive cranial radiotherapy and continue the trastuzumab treatment $[16,17]$.

Cardiotoxicity is of great concern during long-term use of trastuzumab and cardiac function must be monitored closely.

\section{Acknowledgements}

Formatting of funding sources

This research did not receive any specific grant from funding agencies in the public, commercial, or not-for-profit sectors.

\section{References}

[1] Slamon, D. J., Clark, G. M., Wong, S. G., Levin, W. J., Ullrich, A., and McGuire, W. L. 1987. "Human Breast Cancer: Correlation of Relapse and Survival with Amplification of the HER-2/neu Oncogene.” Science 235 (4785): 177-82.

[2] Fabi, A., Malaguti, P., Vari, S., and Cognetti, F. 2016. "First Line Therapy in HER2 Positive Metastatic Breast Cancer: Is the Mosaic Fully Completed or Are We Missing Additional Pieces?” J Exp Clin Cancer Res 35: 104.

[3] Slamon, D. J., Godolphin, W., Jones, L. A., Holt, J. A., Wong, S. G., Keith, D. E., et al. 1989. "Studies of the HER-2/neu Proto-oncogene in Human Breast and Ovarian Cancer.” Science 244: 707-12.

[4] Slamon, D. J., Leyland-Jones, B., Shak, S., Hank, F., Virginia, P., Alex, B., et al. 2001. "Use of Chemotherapy plus a Monoclonal Antibody against HER2 for Metastatic Breast Cancer that Overexpresses HER2.” N Engl J Med 344: 783-92.

[5] Burstein, H. J., Lieberman, G., Slamon, D. J., Winer, E. P., and Klein, P. 2005. "Isolated Central Nervous System Metastases in Patients with HER2 Overexpressing Advanced Breast Cancer Treated with First-line Trastuzumab-based Therapy.” Ann Oncol 16: 1772-7.

[6] Lower, E. E., Drosick, D. R., Blau, R., Brennan, L., Danneman, W., and Hawley, D. K. 2003. "Increased Rate of Brain Metastasis with Trastuzumab Therapy not Associated with Impaired Survival.” Clin Breast Cancer 4: 114-9.

[7] Badulescu, F., Badulescu, A., Paul, D., Popescu, C. F., and Florescu, C. 2014. "More than 9 Years of Continuous Trastuzumab Treatment in Metastatic Breast Cancer without Cardiac Toxicity: A Case Report and Literature Review.” Onco Targets Ther 7: 1911-7.

[8] Murthy, P., Kidwell, K. M., Schott, A. F., Merajver, S. D., 
Griggs, J. J., and Smerage, J. D. 2016. "Clinical Predictors of Long-term Survival in HER2-positive Metastatic Breast Cancer.” Breast Cancer Res Treat 155: 589-95.

[9] Slimane, K., Andre, F., Delaloge, S., Dunant, A., Perez, A., Grenier, J., et al. 2004. "Risk Factors for Brain Relapse in Patients with Metastatic Breast Cancer.” Ann Oncol 15: 1640-04.

[10] Gabos, Z., Sinha, R., Hanson, J., Chauhan, N., Hugh, J., Mackey, J. R., and Abdulkarim, B. 2006. "Significance of Human Epidermal Growth Factor Receptor Positivity for the Development of Brain Metastasis after Newly Diagnosed Breast Cancer.” J Clin Oncol 24: 5658-63.

[11] Gaspar, L., Scott, C., Rotman, M., Asbell, S., Phillips, T., Wasserman, T., et al. 1997. "RPA (Recursive Partitioning Analysis) of Prognostic Factors in the Three RTOG (Radation Therapy Oncology Group) Brain Metastases Trials.” Int J Radiast Oncol Biol Phys 37: 745-51.

[12] Kirsch, D. G., Ledezma, C. J., Mathews, C. S., Bhan, A. K., Ancukiewicz, M., Hochberg, F. H., et al. 2005. "Survival after Brain Metastases from Breast Cancer in the Trastuzumab Era.” J Clin Oncol 23: 2114-6.
[13] Park, I. H., Ro, J., Lee, K. S., Nam, B. H., Kwon, Y., and Shin, K. H. 2009. "Treatment beyond Brain Progression in HER2-Positive Metastatic Breast Cancer.” Ann Oncol 20: 56-62.

[14] Seidman, A., Hudis, C., Pierri, M. K., Shak, S., Paton, V., Ashby, M., et al. 2002. "Cardiac Dysfunction in the Trastuzumab Clinical Trials Experience.” J Clin Oncol 20: 1215-21.

[15] Advani, P. P., Ballman, K. V., Dockter, T. J., Colon-Otero, G., and Perez, E. A. 2016 "Long-term Cardiac Safety Analysis of NCCTG N9831 (Alliance) Adjuvant Trastuzumab Trial.” J Clin Oncol 34: 581-7.

[16] John, S., Anna, D., and Nicolas, T. 2010. "Sustained Complete Remission of Human Epidermal Growth Factor Receptor 2-positive Metastatic Breast Cancer in the Liver Long-term Trastuzumab Maintenance Therapy in a Woman: A Case Report.” J Med Case Rep 4: 401.

[17] Metro, G., Sperduti, I., Russillo, M., Milella, M., Cognetti, F., and Fabi, A. 2007. "Clinical Utility of Continuing Trastuzumab beyond Brain Progression in HER2-positive Metastatic Breast Cancer.” Oncologist 12: 1467-71. 